\title{
Galcanezumab Provides Consistent Efficacy Throughout the Dosing Interval Among Patients with Episodic and Chronic Migraine: A Post Hoc Analysis
}

\author{
Patricia Pozo-Rosich • Karen H. Samaan (D) - Todd J. Schwedt • \\ Robert A. Nicholson · Mallikarjuna Rettiganti · Eric M. Pearlman
}

Received: December 9, 2020 / Accepted: March 12, 2021 / Published online: May 5, 2021

(C) The Author(s) 2021

\begin{abstract}
Introduction: The consistency of the treatment effect of galcanezumab throughout the dosing interval is examined in patients with episodic and chronic migraine.

Methods: This study was a post hoc analysis of clinical trial data from episodic (EVOLVE-1; EVOLVE-2; both 6-month duration) and chronic (REGAIN; 3-month duration) migraine double-blind trials evaluating the efficacy of a once-monthly injection of galcanezumab $120 \mathrm{mg}$ relative to placebo. Adults with episodic (placebo, $n=894$; galcanezumab, $n=444$ ) or chronic migraine (placebo, $n=558$; galcanezumab, $n=278$ ) were included. Mean change from baseline in weekly migraine
\end{abstract}

\footnotetext{
P. Pozo-Rosich

Headache Unit, Neurology Department, Vall d'Hebron University Hospital, Barcelona, Spain

P. Pozo-Rosich

Headache and Neurological Pain Research Group, Department de Medicine, Vall d'Hebron Research Institute, Universitat Autònoma de Barcelona, Barcelona, Spain

K. H. Samaan $(\varangle) \cdot$ R. A. Nicholson · M. Rettiganti . E. M. Pearlman

Eli Lilly and Company, LTC-South, Indianapolis, IN, USA

e-mail: karen.samaan@lilly.com

T. J. Schwedt

Mayo Clinic, Scottsdale, AZ, USA
}

headache days, averaged across all months for each week of the dosing interval, was compared between groups and within the galcanezumab group during weeks 1 and 4 . Additional analyses examined the mean difference from placebo in weekly migraine headache days and a day-byday analysis.

Results: Weekly migraine headache day reduction was significantly greater with galcanezumab relative to placebo every week $(P<0.001)$ and did not differ during weeks 1 and 4 for those with episodic $(P=0.740)$ or chronic migraine $(P=0.231)$ taking galcanezumab. Estimated probabilities of migraine on day 2 and day 30 did not differ for those with episodic $(P=0.61)$ or chronic migraine $(P=0.616)$ taking galcanezumab.

Conclusion: This analysis demonstrates once monthly galcanezumab exhibits consistent efficacy throughout the dosing interval among the population of patients with migraine in three clinical trials evaluating the efficacy of galcanezumab. There is no evidence from these trials that the effect of galcanezumab "wears off" at the end of the dosing interval.

Trial Registration: ClinicalTrials.gov identifier: EVOLVE-1 (NCT02614183); EVOLVE-2 (NCT02614196); REGAIN (NCT02614261).

Keywords: Chronic; Efficacy; Episodic; Galcanezumab; Migraine; Wear off 


\section{Key Summary Points}

Migraine is a neurologic disease that is a major cause of diminished quality of life and years lost to disability. A lack of consistent response to preventive treatment may contribute to patient noncompliance and early discontinuation of preventive therapy

This post-hoc analysis examined the consistency of the treatment effect of galcanezumab throughout the dosing interval in patients with chronic and episodic migraine

Compared to placebo, patients treated with galcanezumab had greater reduction in weekly migraine headache days every week over 3 months in patients with chronic migraine and 6 months in patients with episodic migraine

During the dosing interval, there was no difference in weekly migraine headache days when the first week was compared to the fourth week, and the estimated probability of having a migraine headache on days 2 and 30 were not different

Among the population of patients in the phase 3 studies, galcanezumab $120 \mathrm{mg}$ once monthly (with a $240 \mathrm{mg}$ loading dose) exhibits consistent efficacy throughout the dosing interval

\section{DIGITAL FEATURES}

This article is published with digital features, including a summary slide, to facilitate understanding of the article. To view digital features for this article go to https://doi.org/10.6084/ m9.figshare.14195273.

\section{INTRODUCTION}

Migraine is a neurologic disease that affects about $12 \%$ of the population worldwide and is a major cause of diminished quality of life and years lost to disability $[1,2]$. Despite a large percentage of patients meeting criteria to receive preventive treatment for migraine $[2,3]$, only a fraction of prevention-eligible patients with migraine receive appropriate treatment $[4,5]$. Among these patients, a lack of consistent response to treatment may contribute to patient non-compliance and early discontinuation of preventive therapy $[6,7]$.

Galcanezumab, a humanized monoclonal antibody that binds to the calcitonin gene-related peptide (CGRP) ligand and blocks its binding to the CGRP receptor [8], is approved for the preventive treatment of migraine in adults. The efficacy of galcanezumab $120 \mathrm{mg}$ administered once monthly (with an initial loading dose of $240 \mathrm{mg}$ ) has been established for the preventive treatment of episodic (EVOLVE-1 and EVOLVE-2 studies) and chronic (REGAIN study) migraine in three phase 3 studies where galcanezumab significantly reduced the number of monthly migraine headache days compared to placebo [9-11].

In patients with episodic migraine, galcanezumab has demonstrated a significant difference relative to placebo as early as the first day after treatment and a superiority in $\geq 50 \%$ reduction in migraine headache days as early as week 1 [12]. Although a clinically meaningful persistence of efficacy with galcanezumab in the treatment of episodic and chronic migraine has been demonstrated across consecutive months [13], the consistency of galcanezumab efficacy within the monthly dosing interval has not been investigated beyond the first month.

The objective of this post hoc analysis was to examine the consistency of treatment effect of galcanezumab $120 \mathrm{mg}$ once monthly (with an initial loading dose of $240 \mathrm{mg}$ ) throughout the dosing interval. 


\section{METHODS}

In this post hoc analysis of three phase 3 , randomized, double-blind, placebo-controlled studies, 1338 adult patients with episodic migraine (placebo, $n=894$; galcanezumab $120 \mathrm{mg}, n=444)$ and 836 adult patients with chronic migraine (placebo, $n=558$; galcanezumab $120 \mathrm{mg}, n=278$ ) were included. Data from the two episodic migraine studies were pooled, and the chronic migraine study was analyzed separately. The pooled studies, EVOLVE-1 (NCT02614183) [9] and EVOLVE-2 (NCT02614196) [10], were designed to examine whether galcanezumab dosed at $120 \mathrm{mg}$ per month with a 240-mg loading dose or dosed at $240 \mathrm{mg}$ per month was superior to placebo in the preventive treatment of episodic migraine. In EVOLVE-1, 858 randomized patients received at least 1 dose of study drug; 433, 213, and 212 patients received placebo, galcanezumab $120 \mathrm{mg}$, and galcanezumab $240 \mathrm{mg}$, respectively. In EVOLVE-2, 915 randomized patients received at least 1 dose of study drug; 461, 231, and 223 patients received placebo, galcanezumab $120 \mathrm{mg}$, and galcanezumab $240 \mathrm{mg}$, respectively. The third study, REGAIN (NCT02614261) [11], was designed to determine whether galcanezumab dosed at $120 \mathrm{mg}$ per month with a 240-mg loading dose or dosed at $240 \mathrm{mg}$ per month was superior to placebo in the preventive treatment of chronic migraine. In REGAIN, 1113 randomized patients received at least 1 dose of study drug; 558, 278, and 277 patients received placebo, galcanezumab $120 \mathrm{mg}$, and galcanezumab $240 \mathrm{mg}$, respectively. The primary outcome measure for each of these studies was the overall mean change from baseline in monthly migraine headache days. The study protocols were approved by the institutional review board for each study site, and patients provided written informed consent prior to study procedures. More information regarding these trials is available in the primary manuscripts [9-11]. This analysis evaluated the galcanezumab $120 \mathrm{mg}$ and placebo treatment arms of the phase 3 studies.

Patients were between 18 and 65 years, with a diagnosis of migraine as defined by the
International Classification of Headache Disorders-3rd edition, beta version [14]. for at least 1 year prior to enrollment and with migraine onset prior to age 50. Patients in the episodic migraine studies had 4-14 migraine headache days per month with an average of no less than 2 migraine attacks per month during the previous 3 months. In the chronic migraine study, patients had at least 15 headache days per month, of which 8 or more had migraine features, with at least 1 or more headache-free days per month for 3 months prior to enrollment.

Full exclusion criteria for these studies are described in the primary manuscripts [9-11]. Of note, patients were excluded from the studies if three or more classes of adequately dosed migraine preventive treatments had failed to provide adequate efficacy.

\section{Statistical Analyses}

The primary objective of the statistical analysis was to determine the consistency of the treatment effect of galcanezumab during each week of the dosing interval, starting with the week immediately after dosing (week 1) and ending with the week immediately prior to the next dose (week 4). The analysis aimed to determine whether the effect of galcanezumab remained consistent throughout the month, starting from the first day after dosing to the day(s) prior to the next dose. These analyses were done for patients in the episodic (pooled data from EVOLVE-1 and EVOLVE-2) and chronic migraine (REGAIN) studies. Mean changes from baseline in weekly migraine headache days during each week of the dosing interval averaged across all months were estimated and compared between the galcanezumab and placebo groups using a mixed-model repeated measures (MMRM) analysis. This model included the following fixed effects variables: baseline migraine headache days, treatment, week, month, study (for episodic only), pooled region/country (nested within study), and the treatment-by-week and baseline-by-week interaction effects. The probability of having a migraine over each day within the month, from day 2 (first day after dosing) through day 30, 
was modeled using a generalized linear mixedeffects model. This model included the following fixed effects variables: baseline migraine headache days, treatment, day, month, study (for episodic data only), and treatment-by-day interaction effect. All multilevel models included patient-level random effects for intercept, week/day, and month variables using a variance components correlation structure to account for the correlation introduced because of repeated measures on each patient measured on each day of each month.

Consistency of treatment effect throughout the dosing interval was evaluated in two ways. First, the treatment-effect difference of galcanezumab relative to placebo was estimated at the weekly or day-by-day level and averaged across all months. This allowed evaluation of whether the treatment effect of galcanezumab later in the dosing interval, relative to placebo, was consistent with its effect early in the dosing interval. Second, within-treatment-group differences were compared. This allowed evaluation of whether the effect of galcanezumab remained consistent later in the dosing interval relative to earlier in the dosing interval. For the weekly analysis, mean weekly migraine headache days were compared between the first and last weeks within each treatment group. For the daily analysis, the estimated probabilities of having a migraine on day 2 (first day after administration) and day 30 were compared to evaluate consistency of effect. These comparisons were done within the context of the multilevel models using contrasts for the leastsquares (LS) means for the treatment-by-period (day or week) interaction effect.

\section{General Considerations}

Baseline demographics were summarized using summary statistics, which included means and standard deviations (SDs) for continuous variables and frequencies and percentages for categorical variables. Effects from linear mixedeffects models were presented using the differences in LS means, whereas effects from generalized linear mixed-effects models were presented as odds ratios (ORs) along with 95\% confidence intervals (CIs). All analyses were conducted using the software SAS ${ }^{\circledR}$ Enterprise
Guide version 7.1. All statistical tests conducted were two-sided, assuming a significance level of $5 \%$. All analyses conducted were post hoc in nature, and thus results should be considered exploratory.

\section{RESULTS}

\section{Baseline Characteristics}

There were no significant differences in baseline characteristics between the patient groups randomized to galcanezumab or to placebo in the episodic or chronic migraine studies, with the exception of age (mean age [SD]: galcanezumab 120 -mg group $=39.7$ [11.9] years, placebo group $=41.6[12.1]$ years; $P<0.05)$. At baseline, prior to randomization, there were no significant differences between the galcanezumab and placebo groups in the number of migraine headache days per week or per month (Table 1). In the episodic trials, the average weekly migraine headache days was $2.1 \pm 0.7$ days, and in the chronic trial, patients treated with galcanezumab averaged $4.5 \pm 1$ weekly migraine headache days versus $4.6 \pm 1.1$ days in the placebo group.

\section{Consistency of Treatment Effect}

\section{Episodic Migraine}

In the weekly analysis of the pooled episodic studies (Fig. 1), the mean reduction from baseline in weekly migraine headache days, averaged across all 6 months, was significantly greater for the galcanezumab group relative to the placebo group in each of the 4 weeks $(P<0.001)$. The mean differences $(95 \% \mathrm{CI})$ in change from baseline of weekly migraine headache days between the galcanezumab group and placebo group were $-0.49(-0.59$, $-0.39)$ in weeks 1 and $2,-0.44(-0.54$, $-0.34)$ in week 3 , and $-0.45(-0.55,-0.35)$ in week 4 . The mean treatment differences in weekly migraine headache days were not significantly different between week 1 and week 4 (difference $=-0.04 ; \quad 95 \% \quad$ CI $-0.12, \quad 0.04$; $P=0.321)$. Treatment-effect estimates were not 
Table 1 Baseline characteristics of phase 3 studies

\begin{tabular}{|c|c|c|c|c|}
\hline & \multicolumn{2}{|c|}{$\begin{array}{l}\text { Episodic Migraine (EVOLVE-1 and } \\
\text { EVOLVE-2) }\end{array}$} & \multicolumn{2}{|c|}{ Chronic Migraine (REGAIN) } \\
\hline & $\begin{array}{l}\text { Placebo, } \\
n=894\end{array}$ & $\begin{array}{l}\text { GMB } \\
120 \mathrm{mg}, \\
n=444\end{array}$ & $\begin{array}{l}\text { Placebo, } \\
n=558\end{array}$ & $\begin{array}{l}\text { GMB } \\
120 \mathrm{mg} \\
n=278\end{array}$ \\
\hline Age, years, mean (SD) & $41.9(11.4)$ & $40.9(11.5)$ & $41.6(12.1)$ & $39.7(11.9)^{*}$ \\
\hline Female, $n(\%)$ & $755(84.5)$ & $378(85.1)$ & $483(86.6)$ & $237(85.3)$ \\
\hline Race, white, $n$ (\%) & $681(76.2)$ & $335(75.5)$ & $432(77.4)$ & $223(80.2)$ \\
\hline North America residence, $n(\%)$ & $657(73.5)$ & $325(73.2)$ & $321(57.5)$ & $161(57.9)$ \\
\hline Years since migraine diagnosis, mean (SD) & $20.5(12.5)$ & $20.5(12.3)$ & $21.9(12.9)$ & $20.4(12.7)$ \\
\hline MIDAS total score, mean (SD) & $33.1(29.3)$ & $31.9(28.0)$ & $68.7(57.4)$ & $62.5(49.5)$ \\
\hline \multicolumn{5}{|c|}{ Number of migraine headache days, mean (SD) } \\
\hline Per month & $9.1(3.0)$ & $9.1(3.0)$ & $19.6(4.6)$ & $19.4(4.3)$ \\
\hline Per week & $2.1(0.7)$ & $2.1(0.7)$ & $4.6(1.1)$ & $4.5(1.0)$ \\
\hline
\end{tabular}

Summary statistics for migraine headache days per week at baseline were obtained by converting migraine headache days per month (30 days) summaries into a 7-day period by dividing them by 30 and multiplying by 7

$G M B$ galcanezumab, $M I D A S$ Migraine Disability Assessment, $n$ number, $S D$ standard deviation

${ }^{*} P<0.05$ versus placebo

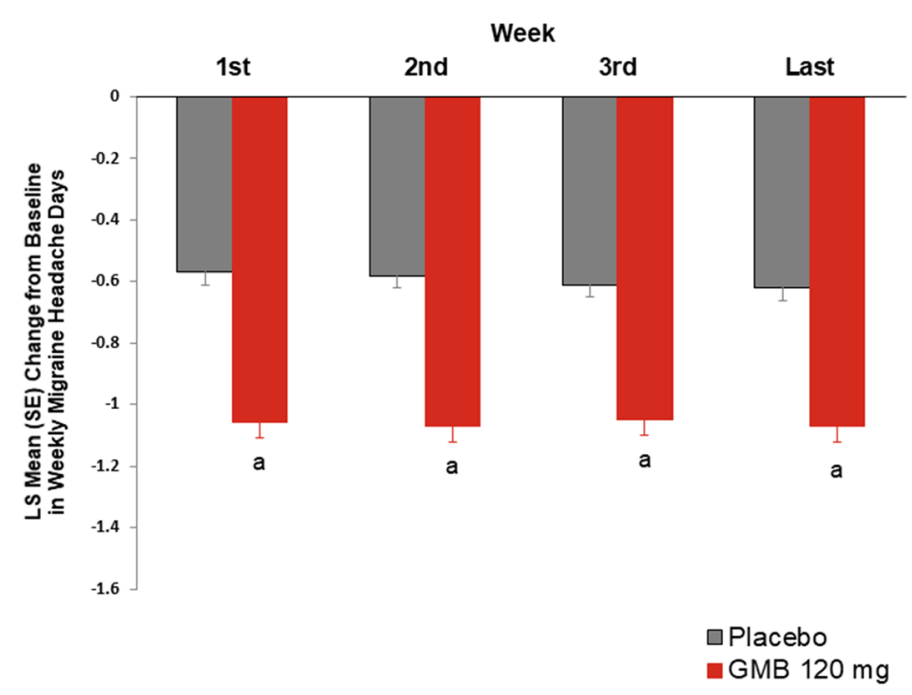

Fig. 1 Episodic migraine: LS mean change from baseline in weekly migraine headache days across all months (EVOLVE-1 and EVOLVE-2 pooled). ${ }^{\mathrm{a}} P<0.001$ versus placebo based on MMRM analysis. Mean changes from baseline for each treatment group were estimated using an MMRM model for repeated measures after adjusting for baseline migraine headache days, week, month, study, pooled region/country (nested within study), and the treatment-by-week and baseline-by-week interaction effects. $G M B$ galcanezumab, $L S$ least-squares, $M M R M$ mixed-model repeated measures, $S E$ standard error 
meaningfully different between other combinations of weeks (results not shown). Within the galcanezumab group, LS mean changes from baseline in weekly migraine headache days during week $1(-1.06)$ and week 4 $(-1.07)$ were not significantly different (difference $=0.01 ; 95 \% \mathrm{CI}-0.05,0.07 ; P=0.740$ ), suggesting the treatment effect of galcanezumab was not "wearing off" during the last week of the dosing interval relative to the first week. The fixed effect of week $(P=0.705)$ and the treatment-by-week interaction effect $(P=0.148)$ from the MMRM model were not statistically significant, suggesting no significant trend in mean changes from baseline in weekly migraine headache days throughout the dosing interval.

In the day-by-day analysis of the pooled episodic studies (Fig. 2), the estimated probability of having a migraine headache on each day, averaged across all 6 months from day 2 through day 30, was significantly lower in the galcanezumab group compared to the placebo group $(P \leq 0.001$ for all days). The galcanezumab group had a significantly lower probability of having a migraine headache compared to the placebo group on day 2 (OR $0.61 ; 95 \%$ CI $0.51,0.72 ; P<0.001)$ and day 30 (OR $0.65 ; 95 \%$ CI $0.51,0.85 ; P=0.001$ ), suggesting the treatment effect relative to placebo is not likely to be different from the beginning to the end of the dosing interval. Within the galcanezumab group, the estimated probabilities of having a migraine headache on day 2 and day 30 were not statistically significantly different (OR 0.94; 95\% CI 0.74, 1.19; $P=0.61$ ), suggesting the treatment effect of galcanezumab was not "wearing off" at the end of the dosing interval relative to the first day following dosing. In addition, the main effect of

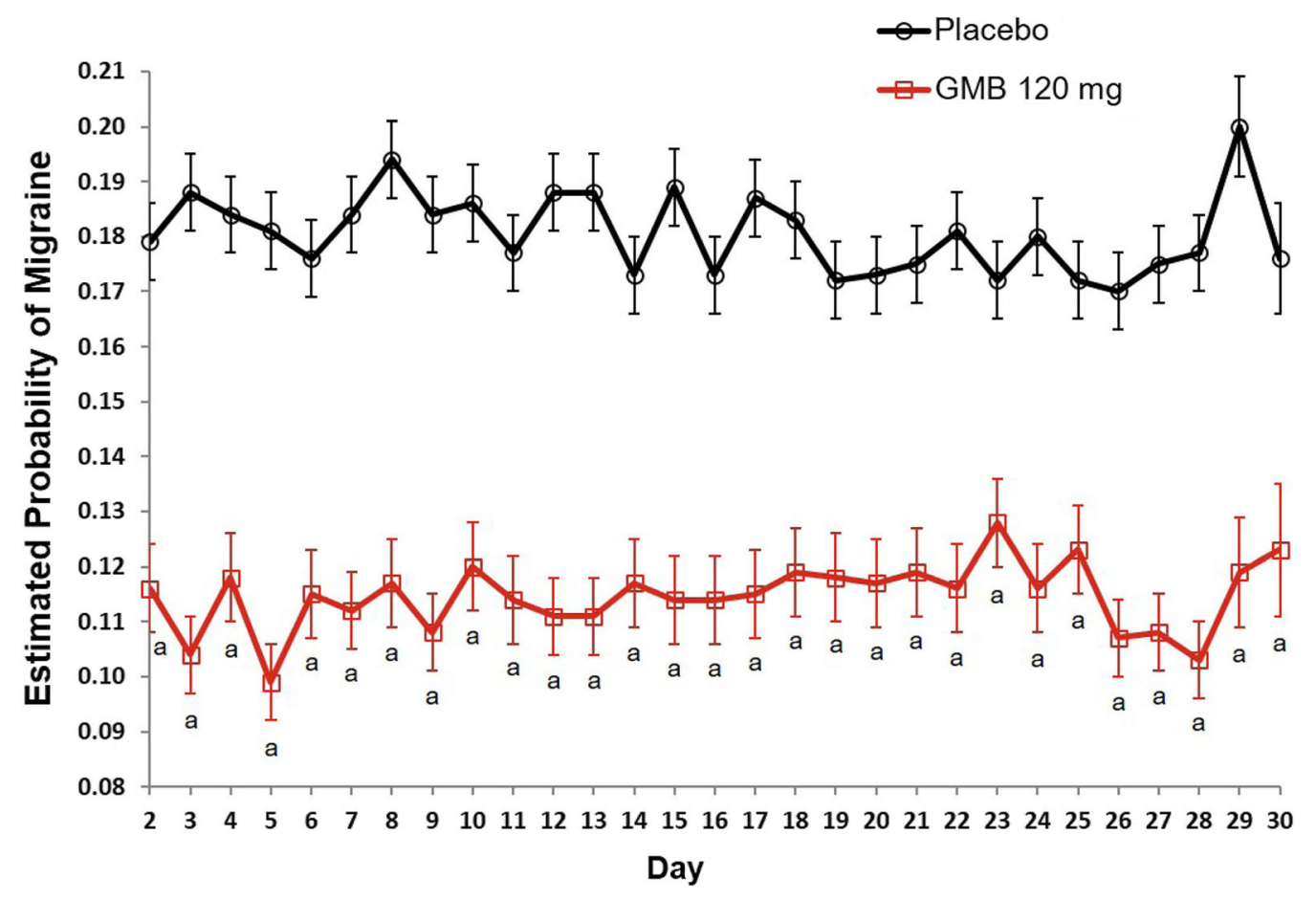

Fig. 2 Episodic migraine: estimated probability of migraine by day averaged across all 6 months (EVOLVE1 and EVOLVE-2 pooled). ${ }^{\mathrm{a}} P \leq 0.001$ versus placebo $(P$ values comparing probability of migraine between the two groups was significant for all days). Probabilities shown for each treatment group were estimated using a generalized linear mixed-effects model for repeated measures adjusting for baseline migraine headache days, day, month, study, and treatment-by-day interaction effect. $G M B$ galcanezumab 
day $(P=0.498)$ and the interaction effect of treatment by day $(P=0.485)$ were not statistically significant, suggesting no evidence of treatment-effect inconsistency during the dosing interval.

\section{Chronic Migraine}

In the weekly analysis of the chronic migraine study (Fig. 3), the mean reduction from baseline in weekly migraine headache days, averaged across all 3 months, was significantly greater for the galcanezumab group compared to the placebo group for each of the 4 weeks $(P<0.001)$. The mean differences (95\% CI) in weekly migraine headache day change from baseline between the galcanezumab group and placebo group were $-0.49(-0.69,-0.28)$ in week 1 , $-0.56(-0.78,-0.34)$ in week $2,-0.52$ $(-0.74,-0.31)$ in week 3 , and $-0.40(-0.62$, $-0.19)$ in week 4 . Treatment differences in weekly migraine headache days were not significantly different between week 1 and week 4 (difference $=-0.08 ; \quad 95 \% \quad$ CI $-0.24, \quad 0.07$; $P=0.301$ ). Treatment-effect estimates were not meaningfully different between other combinations of weeks (results not shown). Within the galcanezumab group, LS mean changes from baseline in weekly migraine headache days during week $1(-1.07)$ and week 4 $(-1.15)$ were not significantly different (difference $=0.08 ; 95 \%$ CI $-0.05,0.20 ; P=0.231$ ), suggesting the effect of galcanezumab did not "wear off" during the last week of the dosing interval relative to the first week. The fixed effect of week $(P=0.162)$ and the treatment-byweek interaction effect $(P=0.403)$ from the MMRM model were not statistically significant, suggesting no significant trend in mean changes from baseline in migraine headache days from week to week within the month.

In the day-by-day analysis of those with chronic migraine (Fig. 4), the estimated probability of having a migraine headache on each day, from day 2 through day 30, averaged across all 3 months, was significantly lower in the galcanezumab group compared to the placebo group on most days $(P<0.05)$ except for days $10(P=0.091), 23(P=0.096), 29(P=0.125)$, and $30(P=0.226)$. The galcanezumab group had a significantly lower probability of having a migraine headache compared to the placebo group on day 2 (OR $0.71 ; 95 \%$ CI $0.56,0.90$; $P=0.005)$ but not on day 30 (OR $0.80 ; 95 \%$ CI

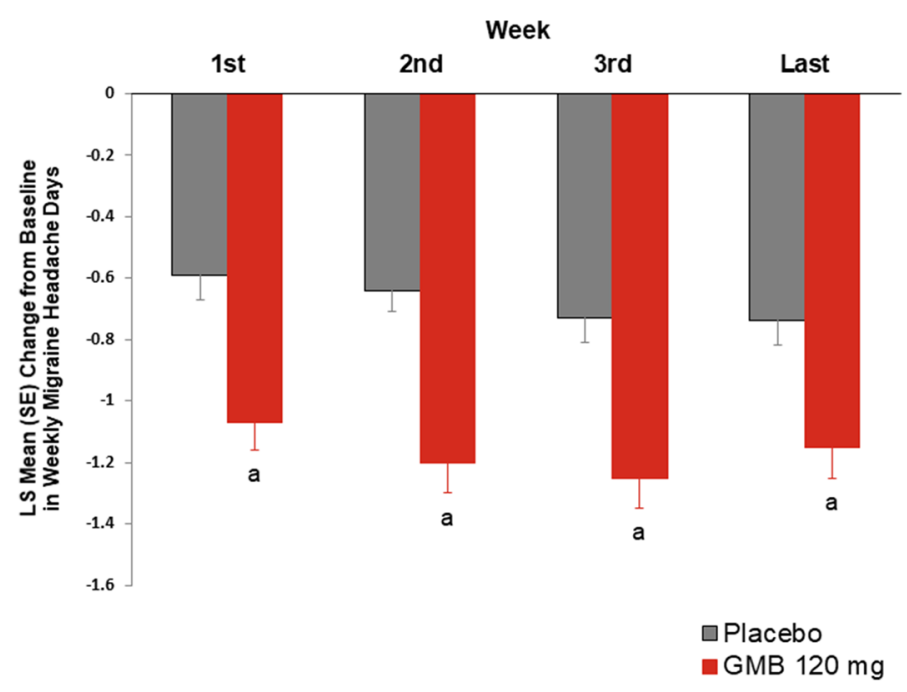

Fig. 3 Chronic migraine: LS mean change from baseline in weekly migraine headache days across all months (REGAIN) for each treatment group. ${ }^{\mathrm{a}} P<0.001$ versus placebo based on MMRM analysis. Mean changes from baseline for each treatment group were estimated using an MMRM model for

repeated measures after adjusting for baseline migraine headache days, week, month, pooled region/country, and the treatment-by-week and baseline-by-week interaction effects. $G M B$ galcanezumab, $L S$ least squares, $M M R M$ mixed-model repeated measures, $S E$ standard error 


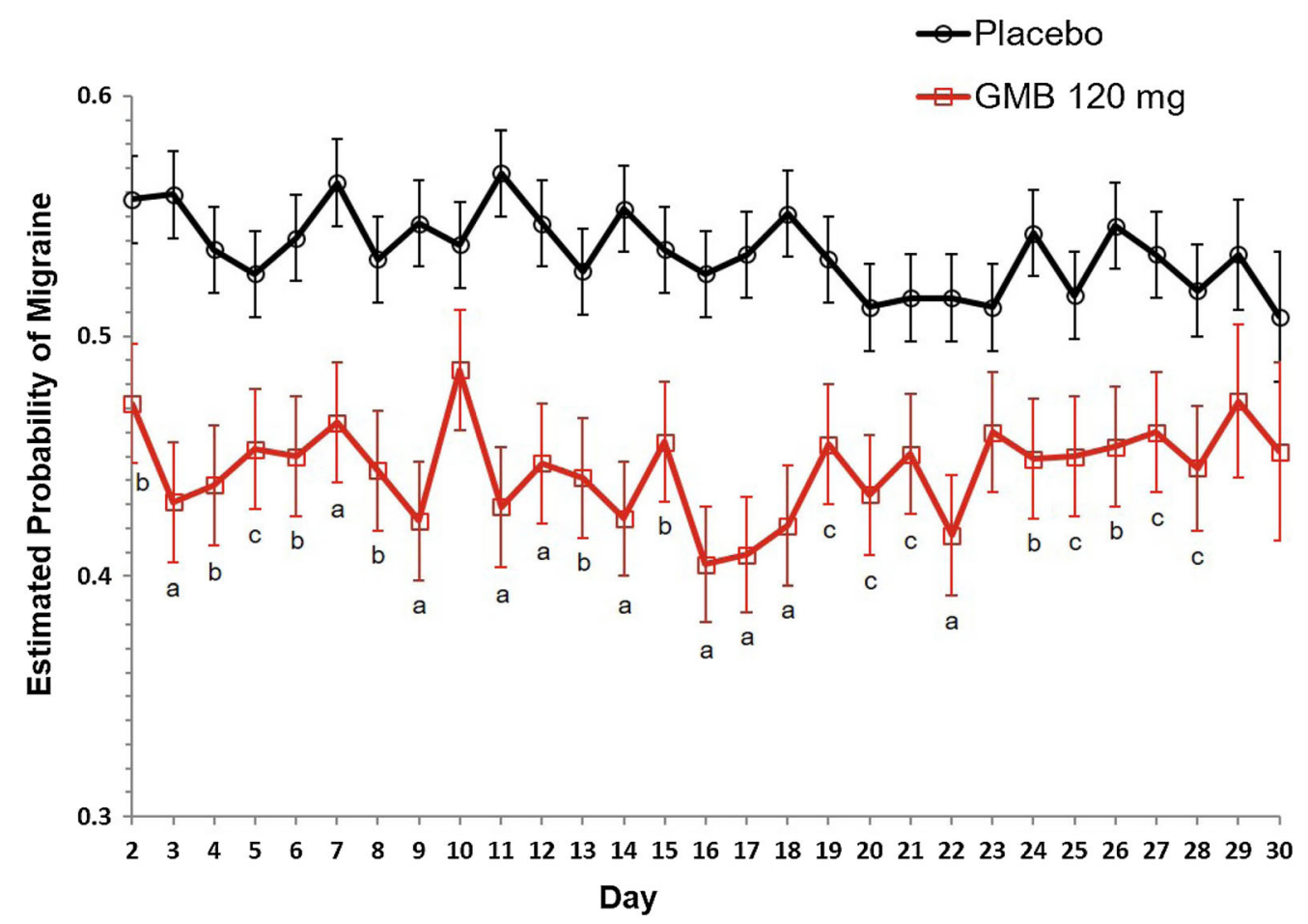

Fig. 4 Chronic migraine: estimated probability of migraine by day averaged across all 3 months (REGAIN). ${ }^{\mathrm{a}} P \leq 0.001$ versus placebo, ${ }^{\mathrm{b}} P \leq 0.010$ versus placebo, ${ }^{\mathrm{c}} P<0.050$ versus placebo. Probabilities shown for each treatment group were estimated using a generalized linear

$0.55,1.15 ; \quad P=0.226)$. Within the galcanezumab group, the estimated probabilities of having a migraine headache on day 2 and day 30 were not statistically significantly different (OR 1.08; 95\% CI 0.80, 1.48; $P=0.616$ ), suggesting the treatment effect of galcanezumab was not "wearing off" at the end of the dosing interval relative to the first day following dosing. The main effect of day $(P=0.389)$ and the interaction effect of treatment-by-day $(P=0.241)$ were not statistically significant, suggesting no evidence that the treatment effect within the galcanezumab group was inconsistent during the dosing interval.

\section{DISCUSSION}

Maintenance of benefit throughout the dosing interval is an important attribute for migraine preventive therapy. Pharmacokinetic studies of mixed-effects model for repeated measures adjusting for baseline migraine headache days, day, month, and treatment-by-day interaction effect. $G M B$ galcanezumab

galcanezumab in healthy patients have shown that galcanezumab has a half-life of 27 days [8] and support treating patients with a oncemonthly subcutaneous injection of galcanezumab. Previous phase 3 studies have shown that galcanezumab at doses of $120 \mathrm{mg}$ and $240 \mathrm{mg}$ significantly reduces the number of mean monthly migraine headache days over 6 months compared to placebo in adults with episodic migraine $[9,10]$ and over 3 months in adults with chronic migraine [11].

Here, we examined whether galcanezumab $120 \mathrm{mg}$ once monthly (with an initial loading dose of $240 \mathrm{mg}$ ) maintained consistent efficacy during the dosing interval in patients with episodic or chronic migraine. Clinical trials evaluating the reduction of migraine headache days in response to a treatment typically use longer durations of time (e.g., months rather than weeks or days) to evaluate treatment efficacy because the natural variation of migraine 
headache in individuals may impact outcomes when comparing treatment to placebo effect. This post hoc analysis attempted to mitigate this variability by averaging data across all months of the phase 3 trials. We evaluated mean reduction of weekly migraine headache days and the day-to-day likelihood of having a migraine headache to determine whether the population of those taking galcanezumab showed a consistent response from the first to the last day of the dosing interval.

This post hoc analysis demonstrates that efficacy of galcanezumab remains consistent throughout the monthly dosing interval for both chronic and episodic migraine. The mean reduction from baseline of weekly migraine headache days averaged across all months was significantly greater in the galcanezumab group compared to the placebo group, and the change in average weekly migraine headache days among those in the galcanezumab group was consistent from the first week to the last week of the dosing interval. These findings suggest that, at a treatment-population level, the effect of galcanezumab remains consistent throughout the dosing interval (i.e., does not "wear off") for those with episodic or chronic migraine.

In the day-by-day analysis, the results among those with episodic migraine show a similar pattern to the weekly data, with the likelihood of having a migraine headache lower among those in the galcanezumab group relative to placebo for all days of the dosing interval and no difference in the likelihood of having a migraine headache within the galcanezumab group at day 2 and day 30. Among those with chronic migraine in the galcanezumab group, there were also no differences in the likelihood of having a migraine headache at day 2 and day 30 of the dosing interval. When comparing the day-to-day likelihood of having a migraine headache between the galcanezumab and placebo groups, the differences were significant at 25 of 29 days. Possible reasons for the lack of statistical significance on other days include the smaller sample size in the chronic study and a smaller sample size reporting headache days towards the end of the month, which could lead to higher variability. In addition, the burden of diary entry (participant fatigue) during the trial may be more likely to occur near the end of the month among patients with higher headache day frequency $[15,16]$. This may be especially relevant for the placebo group who had more headache days to report throughout the month. Finally, the absence of statistical significance does not rule out a clinically meaningful effect. This may help substantiate the message that the effect of galcanezumab does not diminish at the end of the dosing interval relative to the beginning of the dosing interval for those with chronic migraine.

The strengths of the study include the large sample size and the complementary methods used to investigate the consistency of effect. These were necessary since there is no standard definition for the loss of effect or "wearing off" during the dosing interval of migraine preventive medication. This analysis was performed on a post hoc basis and thus should be considered exploratory, so failure to find a statistically significant difference may not provide conclusive evidence of absence of effect. In addition, the analysis is treatment population based, and it cannot be interpreted that every individual will have a consistent response to galcanezumab throughout the dosing interval every month. It is possible to evaluate consistency of effect at an individual level, but that was not in line with the scope and aims of the current study. Future studies might consider whether individual-level factors (e.g., migraine headache day variability, comorbidities, migraine disease-related, carerelated, sociodemographic, psychographic) influence the consistency of effect throughout the month. Identifying a subset of patients inclined to experience inconsistent effect throughout the month would provide clinicians insight that might influence treatment decisions.

These results demonstrate that galcanezumab has consistent efficacy throughout the monthly dosing interval and there is no indication of "wearing off" or loss of effect for patients with episodic or chronic migraine. These results expand the information available to providers and patients about the expected outcomes when using galcanezumab for the preventive treatment of migraine. 


\section{ACKNOWLEDGEMENTS}

The authors thank the study participants for their involvement in the study.

Funding. This study (design and conduct of the study; collection, management, analysis, and interpretation of the data, preparation of the manuscript, and decision to submit the manuscript for publication) was funded by Eli Lilly and Company, Indianapolis, IN, USA. The journal's Rapid Service and Open Access Fees are also funded by the study sponsor (Eli Lilly and Company, Indianapolis, IN, USA).

Medical writing, editorial, and other assistance. Editorial assistance in the preparation of the manuscript was provided by Antonia Baldo. Medical writing assistance in the preparation of the manuscript was provided by Deborah D'Souza. Support for this assistance was funded by Eli Lilly and Company.

Authorship. All named authors meet the International Committee of Medical Journal Editors (ICMJE) criteria for authorship for this article, take responsibility for the integrity of the work as a whole, and have given their approval for this version to be published.

Prior presentation. This manuscript is based on work that has been previously presented at 19th Congress of International Headache Society, Dublin, Ireland, September 5-8, 2019; 13th Annual Headache Cooperative of the Pacific, California, USA, January 24-25, 2020; Presented at the 2020 American Academy of Neurology Science Highlights Platform (Annual Meeting On Demand 2020), May 18, 2020; Presented at 2020 American Association of Nurse Practitioners (AANPconnect), September 10-December 31, 2020; Spanish Society of Neurology Annual Meeting, Seville, Spain, November 17-21, 2020.

Disclosures. Karen H. Samaan, Robert A. Nicholson, Mallikarjuna Rettiganti, and Eric M. Pearlman are full time employees and minor stockholders of Eli Lilly and Company. Patricia Pozo-Rosich has received honoraria as a consultant and speaker for Allergan, Amgen, Almirall, Biohaven, Chiesi, Eli Lilly and Company, Medscape, Novartis, and Teva Pharmaceuticals. Her research group has received research grants from Allergan, Novartis, Instituto de Salud Carlos III, La Caixa Foundation, EraNet Neuron, Migraine Research Foundation, and International Headache Society, and has received funding for clinical trials from Alder, Boehringer Ingelheim, MSD, electroCore, Eli Lilly and Company, Janssen Cilag, Novartis, and Teva. She is a trustee member of the board of the International Headache Society, a member of the Council of the European Headache Federation, a member of the Scientific Advisory Committee of the Migraine Research Foundation and Lilly Spain Foundation. She is on the editorial board of Revista de Neurologia. She is an editor for Cephalalgia, Headache, Frontiers in Neurology, Neurologia and is on the advisory Committee of The Journal of Headache and Pain. She is a member of the Clinical Trials Guidelines Committee of the International Headache Society. She has edited the Guidelines for the Diagnosis and Treatment of Headache of the Spanish Neurological Society. She is the founder of www.midolordecabeza.org. She does not own stocks in any pharmaceutical company. Todd J. Schwedt reports research grants from the American Migraine Foundation, Amgen, Henry Jackson Foundation, National Institutes of Health, the Patient Centered Outcomes Research Institute, and United States Department of Defense. He has received personal fees for consulting from Allergan, Alder, Amgen, Biohaven, Cipla, Click Therapeutics, Dr. Reddy's, Eli Lilly and Company, Equinox, Ipsen Bioscience, Lundbeck, Novartis, Teva, Weber and Weber, and XoC. He has stock options from Aural Analytics and Nocira, and receives royalties from UpToDate.

Compliance with ethics guidelines. The study protocols were approved by the institutional review board for each study site and patients provided written informed consent prior to study procedures. More information regarding these trials is available in the primary manuscripts [9-11]. 
Data availability. Data Sharing Statement-Lilly provides access to all individual participant data collected during the trial, after anonymization, with the exception of pharmacokinetic or genetic data. Data are available to request 6 months after the indication studied has been approved in the US and EU and after primary publication acceptance, whichever is later. No expiration date of data requests is currently set once data are made available. Access is provided after a proposal has been approved by an independent review committee identified for this purpose and after receipt of a signed data sharing agreement. Data and documents, including the study protocol, statistical analysis plan, clinical study report, blank or annotated case report forms, will be provided in a secure data sharing environment. For details on submitting a request, see the instructions provided at www.vivli.org.

Open Access. This article is licensed under a Creative Commons Attribution-NonCommercial 4.0 International License, which permits any non-commercial use, sharing, adaptation, distribution and reproduction in any medium or format, as long as you give appropriate credit to the original author(s) and the source, provide a link to the Creative Commons licence, and indicate if changes were made. The images or other third party material in this article are included in the article's Creative Commons licence, unless indicated otherwise in a credit line to the material. If material is not included in the article's Creative Commons licence and your intended use is not permitted by statutory regulation or exceeds the permitted use, you will need to obtain permission directly from the copyright holder. To view a copy of this licence, visit http://creativecommons.org/licenses/by$\mathrm{nc} / 4.0 /$.

\section{REFERENCES}

1. Woldeamanuel YW, Cowan RP. Migraine affects 1 in 10 people worldwide featuring recent rise: a systematic review and meta-analysis of communitybased studies involving 6 million participants. J Neurol Sci. 2017;372:307-15.
2. Lipton RB, Bigal ME, Diamond M, Freitag F, Reed ML, Stewart WF, AMPP Advisory Group. Migraine prevalence, disease burden, and the need for preventive therapy. Neurology. 2007;68:343-9.

3. Lipton RB, Araujo AB, Nicholson RA, et al. Patterns of diagnosis, consultation, and treatment of migraine in the US: results of the OVERCOME study. In: 61st Annual Scientific Meeting American Headache Society ${ }^{\circledR} 2019$ Headache. 2019;59:2-3. https://doi.org/10.1111/head.13549.

4. Ashina S, Nicholson RA, Buse DC, et al. Identifying barriers to care-seeking, diagnosis, and preventive medication among those with migraine: results of the OVERCOME study [abstract]. Headache. 2020;60(S1):127-8.

5. Nicholson RA, Lipton RB, Reed ML, et al. Characteristics of individuals with migraine who are eligible for novel CGRP monoclonal antibodies: results of the OVERCOME study [abstract]. Headache. 2020;60(S1):126-7.

6. Hepp Z, Bloudek LM, Varon SF. Systematic review of migraine prophylaxis adherence and persistence. J Manag Care Pharm. 2014;20:22-33.

7. D'Amico D, Tepper SJ. Prophylaxis of migraine: general principles and patient acceptance. Neuropsychiatr Dis Treat. 2008;4:1155-67.

8. EMGALITY (Prescribing Information). Indianapolis, IN: Eli Lilly and Company, 2019. https://pi.lilly. com/us/emgality-uspi.pdf.

9. Stauffer VL, Dodick DW, Zhang Q, Carter JN, Ailani J, Conley RR. Evaluation of galcanezumab for the prevention of episodic migraine: the EVOLVE-1 randomized clinical trial. JAMA Neurol. 2018;75: 1080-8.

10. Skljarevski V, Matharu M, Millen BA, Ossipov MH, Kim B-K, Yang JY. Efficacy and safety of galcanezumab for the prevention of episodic migraine: results of the EVOLVE-2 phase 3 randomized controlled clinical trial. Cephalalgia. 2018;38:1442-54.

11. Detke HC, Goadsby PJ, Wang S, Friedman DI, Selzler KJ, Aurora SK. Galcanezumab in chronic migraine: the randomized, double-blind, placebocontrolled REGAIN study. Neurology. 2018;19: e2211-21.

12. Detke HC, Millen BA, Zhang Q, et al. Rapid onset of effect of galcanezumab for the prevention of episodic migraine: analysis of the EVOLVE studies. Headache. 2020;60:348-59.

13. Förderreuther S, Zhang Q, Stauffer VL, Aurora SK, Lainez MJA. Preventive effects of galcanezumab in adult patients with episodic or chronic migraine are 
persistent: data from the phase 3, randomized, double-blind, placebo controlled EVOLVE-1, EVOLVE-2, and REGAIN studies. J Headache Pain. 2018;19:121.

14. Headache Classification Committee of the International Headache Society (IHS). The international classification of headache disorders, 3rd edition (beta version). Cephalalgia. 2013;33:629-808.
15. O'Connor S, Hanlon P, O'Donnell CA, Garcia S, Glanville J, Mair FS. Understanding factors affecting patient and public engagement and recruitment to digital health interventions: a systematic review of qualititave studies. BMC Med Inform Decis Mak. 2016;16:120.

16. Reynolds BM, Robles TF, Repetti RL. Measurement reactivity and fatigue effects in daily diary research with families. Dev Psychol. 2016;52:442-56. 